\title{
Metodologia para AVAliação de Impacto Ambiental de MACRÓFITAS EM MESOCOSMOS ${ }^{1}$
}

\author{
Methodology for Assessing the Environmental Impact of Macrophytes on Mesocosms \\ GUIMARÃES, G.L. ${ }^{2}$, FOLONI, L.L. ${ }^{3}$, PITELI, R. ${ }^{4}$ e MARTINS, A.T. ${ }^{5}$
}

\begin{abstract}
RESUMO - Este trabalho teve como objetivo analisar a metodologia para avaliação do impacto ambiental causado pelo uso de herbicidas no controle de macrófitas. O desenho e a construção dos mesocosmos são descritos em detalhes, como também a forma como foram inseridos lâminas para coleta e medida da comunidade perifitica e sedimento para avaliação da comunidade bentônica. O método utilizado foi o de fluxo continuo, e vários parâmetros de qualidade de água foram medidos, além da determinação de resíduos na coluna d'água e no sedimento. A intenção de se utilizar um método de fluxo contínuo foi buscar uma situação mais próxima da realidade, a fim de que os resultados obtidos possam espelhar com maior exatidão os possiveis riscos provenientes do uso de substâncias químicas no controle das macrófitas. Alguns ajustes devem ser feitos nessa metodologia para melhor caracterização dos efluentes e calibração do sistema.
\end{abstract}

Palavras-chave: herbicida, perifito, bentos, resíduos.

\begin{abstract}
This experiment aimed to analyze the methodology applied to evaluate the environmental impact caused by the use of herbicide to control macrophytes. The design and construction of the tanks are described in detail as well as the insertion of blades for periphyton and sediment sampling to evaluate the bentonic comunities. The flow-through method was used and several parameters of water quality were measured and residues detected in the water colunm and sediment. The flow-through methodology was chosen to create a situation closer to reality so that the results obtained could more precisely reflect the likely risks caused by chemical control of macrophytes. Some adjustments need to be made in this methodology for a better characterization of the effluents and calibration of the system.
\end{abstract}

Key words: herbicide, periphyton, bentonic community, and residues.

\section{INTRODUÇÃO}

É cada vez maior a utilização de produtos químicos em ambientes aquáticos, principalmente em situações nas quais macrófitas aquáticas estão presentes; esta presença tem causado diversos problemas, como obstrução de turbinas e grades de proteção, funcionamento anormal de eclusas, danos ao ambiente, entraves à navegação, abrigo de vetores de várias doenças, além de prejudicar atividades de lazer.

O uso de herbicidas no controle dessas plantas, embora ocorra desde o final da década de 60, necessita ser mais bem avaliado, já que tais substâncias químicas provocam impactos diferentes no meio ambiente, devido às suas diferentes características físico-químicas e à toxicidade a organismos não-alvo, bem como

\footnotetext{
1 Recebido para publicação em 25.3.2003 e na forma revisada em 30.6.2003.

2 Eng.-Agr., Dr., Dow AgroSciences Industrial Ltda, R. Alexandre Dumas 1671, 04717-903 São Paulo-SP. ${ }^{3}$ Eng.-Agr., Dr, Prof. Colab. FEAGRI-UNICAMP, Caixa Postal 6011, 13083-970 Campinas-SP. ${ }^{4}$ Eng.-Agr., Dr., Prof. Titular do DDAA-FCAV-UNESP, 14807-000 Jaboticabal,SP. ${ }^{5}$ Eng.-Agr. UNESP, 14807-000 Jaboticabal-SP.
} 
através da residualidade que podem deixar em diferentes matrizes - neste caso, a água e o sedimento.

A grande maioria dos experimentos sobre o controle de macrófitas em ambientes aquáticos utiliza o método estático, como pode ser comprovado nos estudos realizados no Brasil por Neves et al. (2002), avaliando o controle químico do aguapé; Martins et al. (2002), estudando o controle químico de Pistia stratiotes, Eichhornia crassipes e Salvinia molesta em caixas d'água; e Tanaka et al. (2002), na avaliação de herbicidas para o controle de Egeria em laboratório. Nesses estudos foram utilizadas caixas d'água ou caixas plásticas de diferentes dimensões, com solos diferentes, mantidos a pleno sol, usando método estático, isto é, em alguns casos apenas a água perdida por evaporação era reposta.

Em outras partes do mundo, os estudos visando avaliar impactos no controle de plantas aquáticas ou monitorar o uso de herbicidas aplicaram métodos estáticos, com o uso de potes ou baldes plásticos, em casa de vegetação, com reposição da água perdida por evaporação ou, então, utilizando parcelas experimentais dentro da própria área onde o herbicida estava sendo utilizado; isso pode ser comprovado nos estudos efetuados por Hooper (1958), Gangstad $\&$ Averitt (1972), Getsinger (1985), Getsinger \& Westerdahl (1986) e Gutierrez et al. (1996).

Crosland et al. (1991) avaliaram um modelo artificial, ao ar livre, para simular condições ambientais naturais, num estudo para determinar limites de concentrações tóxicas de produtos químicos em ambientes aquáticos; o método era de fluxo contínuo e parcialmente contínuo com parcial recirculação do sistema, que consistia de seis riachos artificiais. Os pesquisadores avaliaram vários parâmetros de qualidade da água, bem como organismos do perifito e do sedimento.

\section{MATERIAL E MÉTODOS}

O presente ensaio foi conduzido na área experimental do Laboratório Giorgio De Marinis, do Departamento de Biologia Aplicada à Agropecuária da Faculdade de Ciências Agrárias e Veterinárias, UNESP, campus de Jaboticabal.
Foram utilizados 20 mesocosmos (definidos como ecossistemas experimentais ao ar livre por Odum, 1985), constituídos por tanques de concreto com $1,86 \mathrm{~m}$ de diâmetro e $0,40 \mathrm{~m}$ de profundidade, acomodando um volume de aproximadamente $1.080 \mathrm{~L}$, que apresentam entrada e saída da água pela superficie (Figuras 1, 2 e 3). A água que abasteceu os mesocosmos era proveniente de um poço cisterna e foi fornecida em quantidade suficiente para uma vazão média de 2,73 $\mathrm{mL} \mathrm{s}^{-1}$, resultando em um período de renovação do volume d'água de 4,7 dias. A água que sai desses tanques, antes de ir para a rede de esgoto, passa por um sistema de carvão ativado, tirando, assim, qualquer risco de contaminação. Esse tempo de renovação de água reflete aproximadamente a condição do reservatório de Santana (Piraí-RJ), onde as macrófitas são abundantes e muito problemáticas; criou-se um sistema dinâmico, um método de fluxo contínuo.

O substrato utilizado como sedimento de fundo do mesocosmo era composto de um quarto de areia, um quarto de solo de barranco, um quarto de solo de várzea e um quarto de composto orgânico - substrato comercial Plantmax. Em cada mesocosmo foi colocada uma camada de $10 \mathrm{~cm}$ de sedimento.

Os tratamentos experimentais constaram de cinco situações de manejo dos mesocosmos, com quatro repetições. Os mesocosmos (considerados parcelas experimentais) foram dispostos num delineamento inteiramente casualizado, com quatro repetições; todos os valores obtidos foram submetidos a testes de análise de variância e teste de Tukey.

Nos mesocosmos colonizados por macrófitas, as espécies presentes não devem ocupar a área total da superficie do tanque; elas devem ocupar a mesma área dentro de um mesocosmo.

No estudo experimental, parte das plantas foi morta por congelamento; este método físico foi escolhido para que as plantas preservassem suas características nutricionais e que apenas os nutrientes pudessem ser liberados no ambiente após o retorno das plantas aos respectivos mesocosmos. A morte das macrófitas por congelamento foi efetuada em dois dias subsequentes; a cada dia metade das plantas do mesocosmo era colocada por 18 horas em congelador à temperatura de $-8{ }^{\circ} \mathrm{C}$. 
Entrada de água
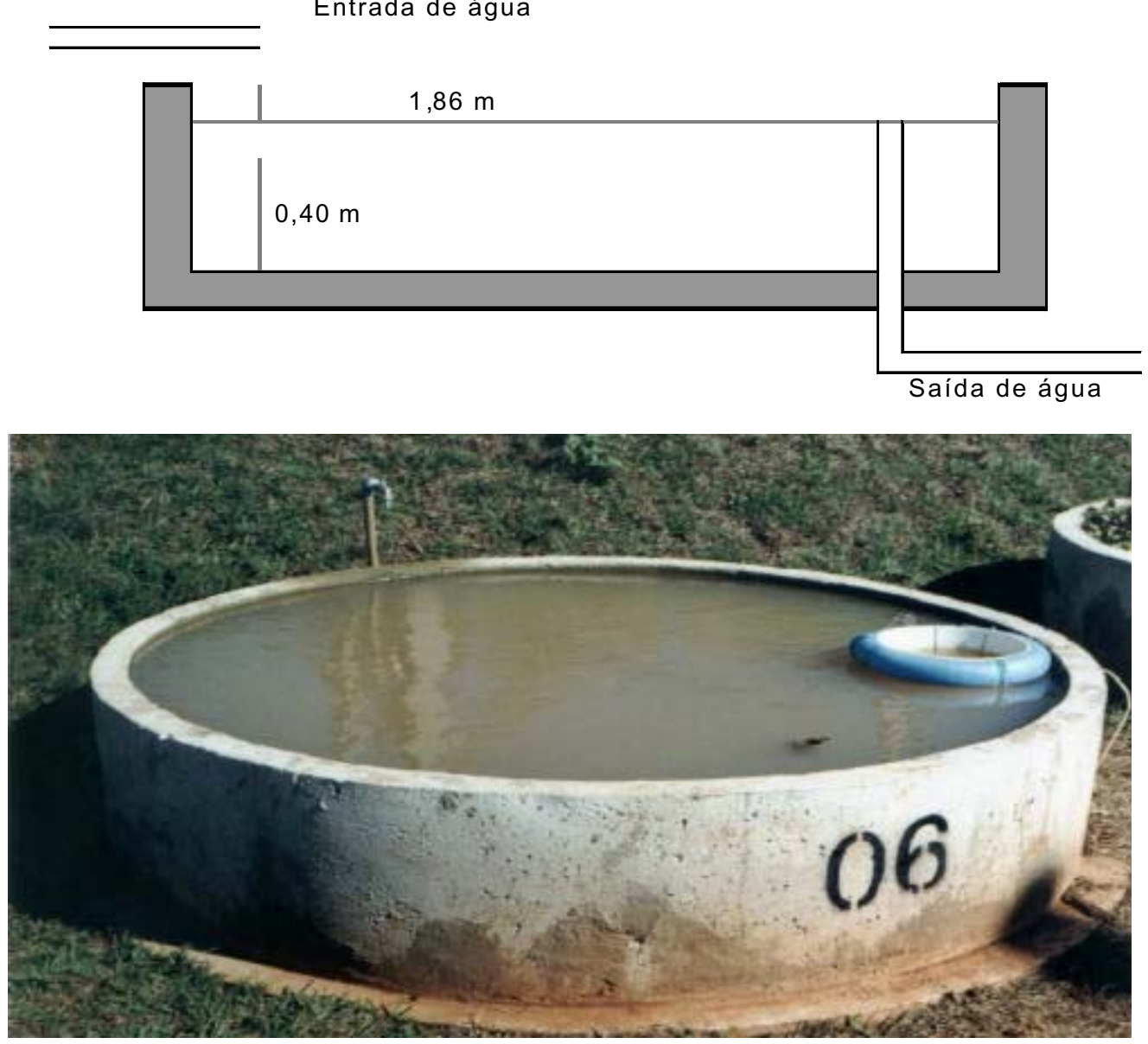

Figura 1 - Representação esquemática e fotografia do mesocosmo utilizado no presente experimento.

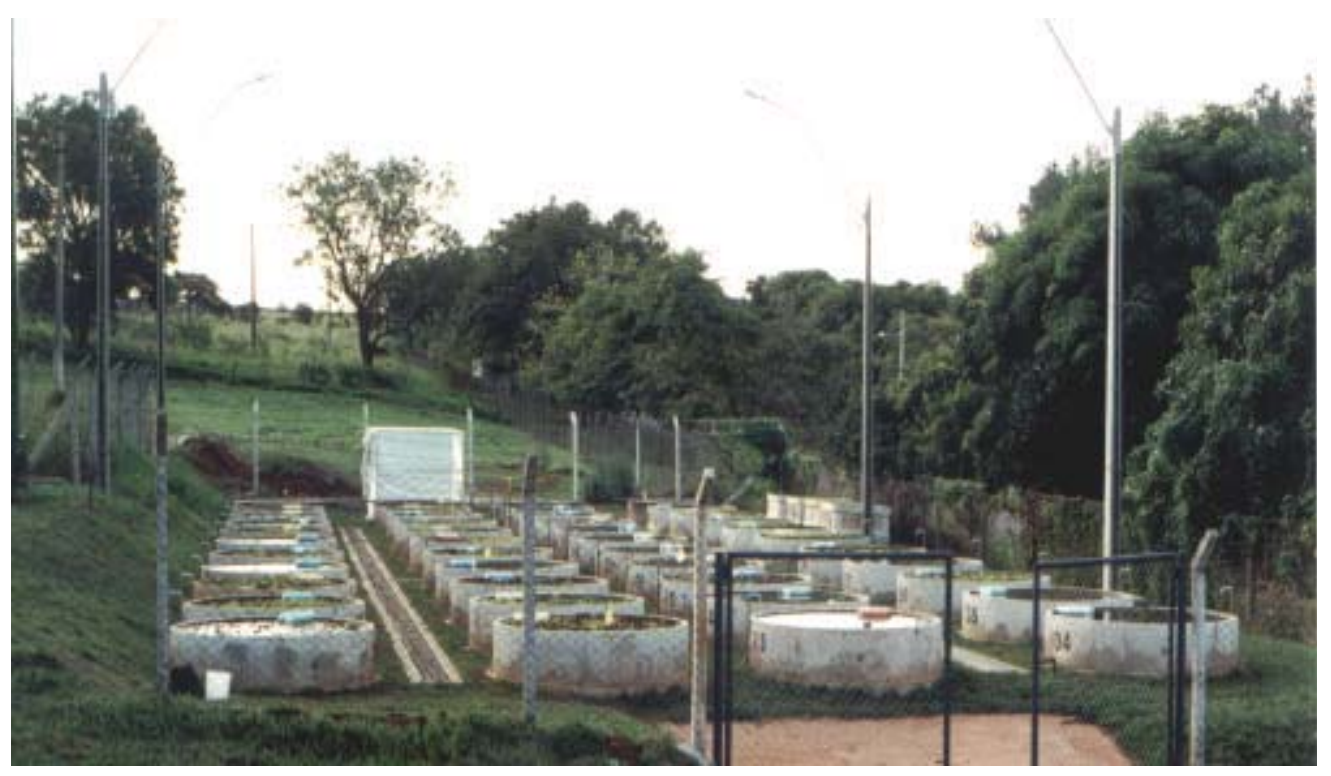

Figura 2 - Vista total da área experimental. 


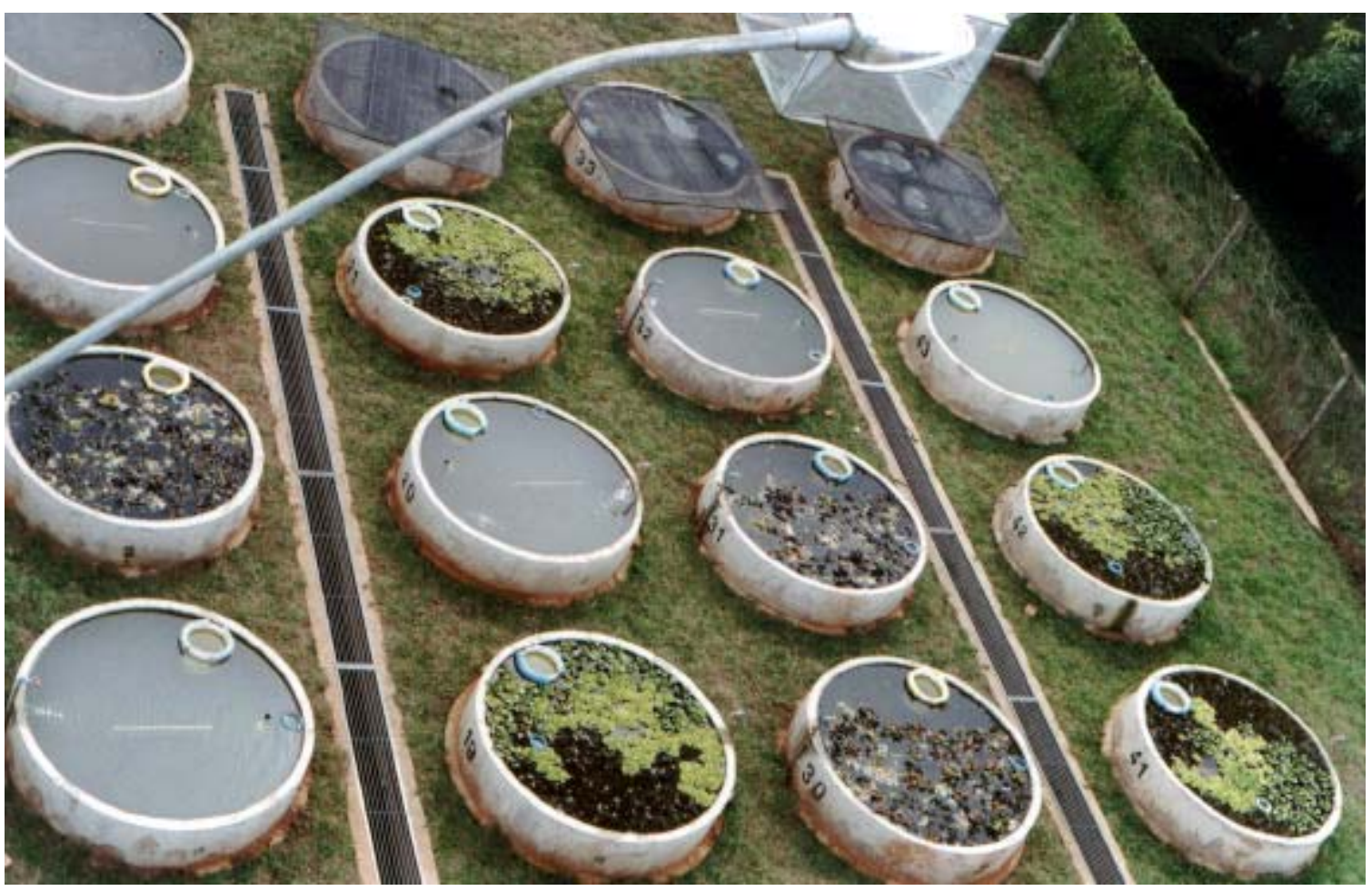

Figura 3 - Vista parcial da área experimental.

O herbicida foi aplicado nos mesocosmos com pulverizador costal à pressão constante (mantida pelo $\mathrm{CO}_{2}$ comprimido). Para evitar que uma deriva da calda do herbicida atingisse outros mesocosmos, durante a aplicação a barra do pulverizador foi colocada dentro de uma estrutura protegida, formada de metal e plástico (Figura 4).

Diariamente, foram efetuadas duas leituras, entre 7 e 7 h30 e entre 17 e 17 h30, utilizando o oximetro YSI 85 da Yellowspring Co para avaliação das seguintes características da água dos mesocosmos: concentração de oxigênio dissolvido $\left(\mathrm{mg} \mathrm{L}^{-1}\right)$, saturação de oxigênio (\%), condutividade elétrica $\left(\mathrm{uS} \mathrm{cm}^{-2}\right) \mathrm{e}$ temperatura $\left({ }^{\circ} \mathrm{C}\right)$. $\mathrm{O} \mathrm{pH}$ da água do mesocosmo também foi avaliado duas vezes por dia, nos mesmos horários, utilizando um peagômetro (Corning pH-10 da "Mite"); a turbidez foi avaliada apenas uma vez ao dia, às 17 horas, através do turbidimetro da Digimed MC-2; a água coletada para as análises foi retirada em torno de $10 \mathrm{~cm}$ de profundidade.

A determinação da clorofila a foi feita utilizando o método do Álcool Etílico, e o cálculo foi feito usando-se a fórmula proposta por Nush (1980).

Para análise do perifito, dez placas de vidro foram colocadas em cada tanque, montadas em estandes de alumínio como suporte (Figura 5); as lâminas $(10 \times 15 \times 0,5 \mathrm{~cm})$ ficaram imersas na profundidade média de $15 \mathrm{~cm}$ abaixo da lâmina d'água. Semanalmente foi coletada uma lâmina de vidro, que foi conservada em vidro "snap cap" de $100 \mathrm{~mL}$, em solução de formol a $8 \%$.

Para a avaliação da comunidade bentônica, foram colocadas, por mesocosmo, dez amostras do sedimento da lagoa marginal do Rio MogiGuaçu, em recipientes plásticos com $500 \mathrm{~g}$ cada um. Uma vez por semana, uma amostra por tanque foi coletada e peneirada em um saco de malha de 0,5 mm, usando água corrente; o material retido foi fixado em formol a $8 \%$ e armazenado em potes plásticos de $300 \mathrm{~mL}$. As comunidades perifitica e bentônica foram avaliadas e classificadas no Laboratório de Limnologia do Departamento de Zoologia do Instituto de Biociências da UNESP, campus de Botucatu. A identificação e a contagem dos 


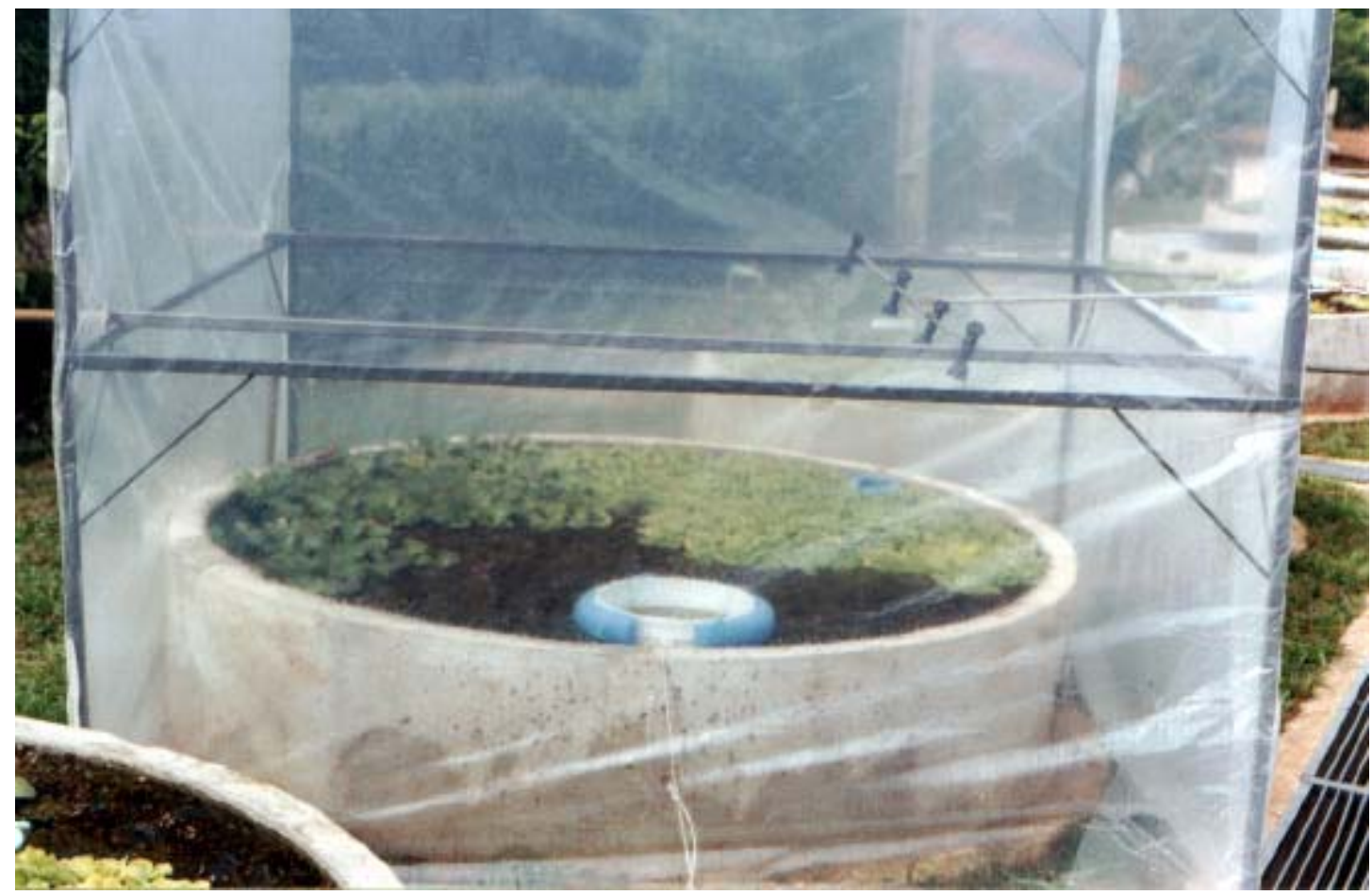

Figura 4 - Detalhe da aplicação do herbicida 2,4-D nos mesocosmos, com a proteção plástica contra deriva.

organismos presentes foram feitas com o uso de um microscópio de imagem invertida (invertoscópio) e câmaras de Sedwick-Rafter com capacidade de $1 \mathrm{~mL}$, com $20 \mathrm{~mm}$ de largura por $50 \mathrm{~mm}$ de comprimento e $1 \mathrm{~mm}$ de profundidade; a identificação foi feita com o apoio das chaves de identificação, e a contagem, através do método da estimativa pelo volume total da amostra, após homogeneização.

Para melhor compreensão dos resultados relacionados com as comunidades perifiticas e bentônicas, foram aplicados índices de diversidade, que usam basicamente dois tipos de informações, como indicado por Coelho (2000): o número de espécies e o número de indivíduos de cada espécie.

Para a determinação de resíduos do produto na água, amostras foram coletadas antes da aplicação e 1, 3, 7, 15, 30, 45 e 60 dias após a aplicação entre 10 e $20 \mathrm{~cm}$ abaixo da lâmina d'água. Essas datas permitem determinar a curva de decaimento do produto e também a meia-vida do herbicida.

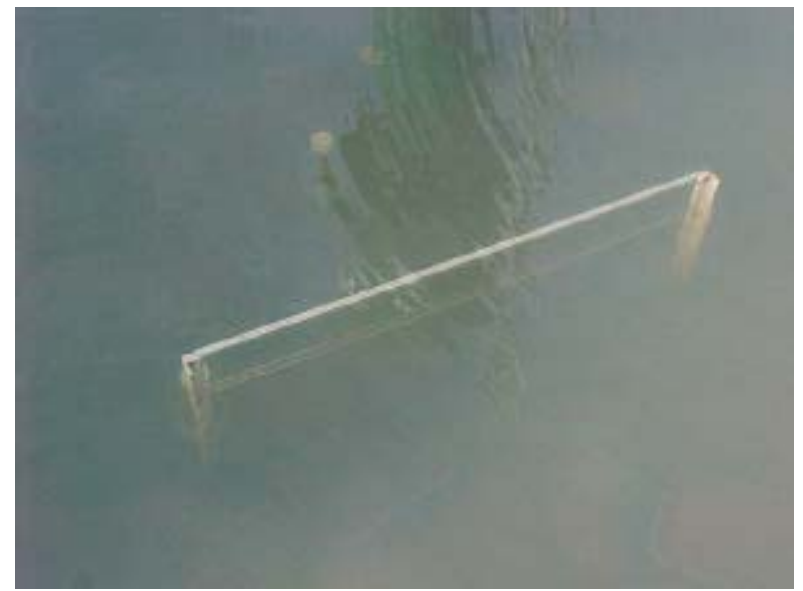

Figura 5 - Estande de perifito instalado no fundo do mesocosmo.

Para determinação dos resíduos de 2,4-D no sedimento do mesocosmo, em cada tanque onde foi aplicado o herbicida foram retiradas amostras do sedimento, antes da aplicação e $1,3,7,15,30,45$ e 60 dias após a aplicação, as quais foram acondicionadas em sacos plásticos. 


\section{RESULTADOS E DISCUSSÃO}

A avaliação do controle de macrófitas por herbicida foi facilmente notada visualmente, comparando-se as parcelas onde o produto foi aplicado com a parcela testemunha.

Em relação aos parâmetros de qualidade de água e clorofila a, comparando os resultados do trabalho com dados obtidos na literatura, em que foi utilizado método estático, pode-se verificar que não ocorreram grandes diferenças; no entanto, é necessário fazer a determinação e caracterização dos efluentes, já que o sistema é de fluxo contínuo e parte do material proveniente da decomposição das plantas mortas foi continuamente sendo retirada do sistema.

As coletas de amostras para todas as determinações foram realizadas de maneira a simular a condição mais real, e esta metodologia mostrou ser muito adequada a esse propósito. Da mesma forma, a maneira como foram inseridas as lâminas para avaliação do perifito e o sedimento para análise da comunidade bentônica torna o método bastante prático e eficiente.

A quantidade de dados obtidos para a determinação e classificação dos organismos aquáticos permite análises estatísticas bastante acuradas e especificas, que podem gerar muitas informações adicionais àquelas pretendidas pelo estudo realizado.

De modo geral, a metodologia utilizada neste estudo de avaliação do impacto ambiental causado pelo controle de macrófitas aquáticas por herbicida mostrou-se altamente satisfatória. Simulando condições próximas do real, os resultados obtidos podem trazer informações valiosas no processo de decisão quanto ao uso de produtos químicos em ambientes aquáticos.

\section{LITERATURA CITADA}

CROSSLAND, N. O. et al. An outdoor artificial stream system designed for ecotoxicological studies. Ecotoxicol. Environ. Safety, v. 22, p. 175-183, 1991.
GANGSTAD, E. O.; AVERITT, W. K. Aquatic plant control program. - Controlled-Release Herbicides. Vicksburg: U.S. Army Engineer, Waterways Experiment Station, 1972. (Technical Report, 1)

GETSINGER, K. D. Chemical control technology development - Evaluation of herbicide / Adjuvant mixtures in flowing water. In: ANNUAL MEETING, AQUATIC PLANT CONTROL PROGRAM, 19., 1985, Vicksburg. Proceedings... Vicksburg: Environmental Laboratory, 1985. p. $115-121$.

GETSINGER, K. D.; WESTERDAHL, H. E. Evaluation of herbicide / Adjuvant mixtures in flowing water (cont.). Aquatic Plant Control Research Program. Vicksburg: U.S. Army Engineer Waterways Experiment Station , 1986. p. 1-23.

GUTIERREZ, R. H. et al. Strategies for waterhyacinth (Eichhornia crassipes) control in Mexico. Hydrobiologia, n. 340, p. 181-185, 1996.

HOOPER, F. N. The effect of applications of pelleted 2,4-D upon the botton fauna of Kent Lake, Michigan. North Center. Weed control Conf. Proc., v. 15, p. 1-41, 1958.

MARTINS, D. et al. Controle químico de Pistia stratiotes, Eichhornia crassipes e Salvinia molesta em caixas d'água. Planta Daninha, v. 20, p. 83-88, 2002. (Edição Especial)

NEVES, T.; FOLONI, L. L.; PITELLI, R. Controle químico do aguapé (Eichhornia crassipes). Planta Daninha, v. 20, p. 89-97, 2002. (Edição Especial)

NUSH, E. Comparison of differents methods for chlorophilla and phaeropigments determination. Arch. Hydrobiol., v. 4, p. 14-36, 1980.

ODUM, E. P. Ecologia. Rio de Janeiro: Interamericana, 1985. $434 \mathrm{p}$.

TANAKA, R. et al. Avaliação de herbicidas para o controle de egéria em laboratório, caixa d'água e represa sem fluxo de água. Planta Daninha, v. 20, p. 73-82, 2002. (Edição Especial) 